\title{
Shedding light on metabotropic glutamate receptors using optogenetics and photopharmacology
}

\author{
Cyril Goudet $^{1 *}$, Xavier Rovira ${ }^{2 *}$ and Amadeu Llebaria ${ }^{3 *}$ \\ ${ }^{1}$ IGF, CNRS, INSERM, Univ. de Montpellier, Montpellier, F-34094 Montpellier, France \\ ${ }^{2}$ Molecular Photopharmacology Research Group, The Tissue Repair and Regeneration \\ Laboratory, University of Vic - Central University of Catalonia, C. de la Laura,13, 08500 Vic, \\ Spain \\ ${ }^{3}$ MCS, Laboratory of Medicinal Chemistry, Institute for Advanced Chemistry of \\ Catalonia (IQAC-CSIC), Barcelona, Spain
}

*Corresponding authors: Cyril Goudet, IGF, 141, rue de la Cardonille, F-34094 Montpellier Cedex 5, France. E-mail: Cyril.goudet@igf.cnrs.fr; Xavier Rovira_Molecular Photopharmacology Research Group, The Tissue Repair and Regeneration Laboratory, University of Vic - Central University of Catalonia, C. de la Laura,13, 08500 Vic, Spain. Email: xavier.rovira@uvic.cat; Amadeu Llebaria, MCS, Laboratory of Medicinal Chemistry, IQAC-CSIC, Jordi Girona 18-24, Barcelona, Spain. E-mail: amadeu.llebaria@iqac.csic.es 


\section{Abstract}

Metabotropic glutamate receptors (mGluRs) are a family of G protein-coupled receptors activated by glutamate, the main excitatory neurotransmitter of the mammalian central nervous system. These receptors are considered as potential therapeutic targets in many neurological diseases but a better understanding of their complex molecular dynamics and of their role in the normal and pathological functioning of the brain is still required. Manipulating mGluRs with high spatial and temporal precision holds great promise for deciphering their physiological and pathological functions. This article reviews several recently developed optogenetic and photopharmacological solutions for the optical control of mGluRs and their applications, from the study of the molecular dynamics of receptor activation to the study of their roles in vivo.

\section{Short title:}

Optogenetics and photopharmacology of mGluRs

\section{Keywords:}

Receptor; Neurotransmitter; Glutamate; Optical control; Optogenetic; Optopharmacology 


\section{Highlights}

- mGluRs can be precisely manipulated by light using photopharmacology or optogenetic strategies.

- Optogenetic pharmacology targets engineered receptors, photopharmacology endogenous ones.

- Optogenetic pharmacology uses photocontrolable ligands binding to genetically modified mGluRs.

- Photopharmacology is based on freely diffusible photoswitchable or photoactivable ligands.

- These optical approaches have proved useful for research in vitro and in vivo. 


\section{Introduction}

Glutamate is the major excitatory neurotransmitter of the mammalian central nervous system (CNS). It activates two categories of receptors: ionotropic and metabotropic glutamate receptors (iGluRs and mGluRs). While iGluRs are ligand-gated ion channels that mediate fast responses to glutamate, mGluRs are class C G-protein coupled receptors (GPCRs) responsible for slower neuromodulatory actions of glutamate.

Eight genes encoding mGluRs have been identified in mammalian genomes. In addition, several mGluR splice variants have been described, most of them altering the intracellular Cterminal tails [1]. Based on sequence homology, signal transduction and pharmacology, mGluRs are subdivided into 3 groups. Group-I mGluRs (mGlu 1,5$)$ are mainly coupled to $\mathrm{Gq}$ and, as such, activate PLC and generate intracellular calcium signals. Both group-II $\left(\mathrm{mGlu}_{2,3}\right)$ and group-III (mGlu $4,6,7,8)$ mGluRs are mainly coupled to Gi/o and can therefore inhibit adenylate cyclase and regulate the activity of various ion channels [2,3].

The different mGluRs are widely distributed throughout the peripheral and central nervous system. In neurons, group I mGluRs are mostly localized at the post-synapse where they upregulate neuronal excitability, whereas group II and III mGluRs are mostly presynaptic receptors that reduce synaptic transmission. mGluRs are not only present in glutamatergic synapses but they have also been observed in other types of synapses, such as dopaminergic and GABAergic synapses. mGluRs have also been detected in astrocytes, oligodendrocytes and microglia (see [4] for review). In addition, mGluRs are distributed outside the CNS, for example in the heart, adrenal glands or lymphocytes (see [5] for review).

mGluRs are complex allosteric machines composed of a seven transmembrane domain (7TMD), common to all GPCRs, connected to a large bilobate extracellular venus-flytrap 
domain (VFT) through a short and rigid cysteine-rich domain (CRD). Moreover, mGluRs are constitutive dimers cross-linked by a disulfide bridge at the level of the VFT and this dimerization is a prerequisite for mGluRs function [6]. Glutamate binds in the cleft between the two lobes of the VFT. The glutamate-binding pocket is well conserved among the different mGluRs, making the design of subtype selective orthosteric ligands challenging, but several successful molecules have been described [2]. Positive, negative and neutral allosteric ligands that bind in the 7TMD have also been identified. Since this binding pocket is more variable than that of glutamate among mGluR subtypes, it has allowed the discovery of subtype selective allosteric ligands of mGluRs [3]. Allosteric modulators targeting the VFT have also been described, such as cations and anions [7-12]. Moreover, mGluRs that were long believed to exclusively form homodimers can also assemble into heterodimers, multiplying the receptor combinations and their subsequent signaling and modulatory mechanisms [3].

Due to their important role in the regulation of synaptic transmission and neuronal excitability, mGluRs are considered potential therapeutic targets for treating neurological disorders, including anxiety, depression, schizophrenia, chronic pain and Parkinson disease (see $[4,13,14]$ for reviews). However, despite the efforts of pharmaceutical companies, there are currently no marketed drugs targeting mGluRs. Drug development and clinical research would benefit from better knowledge of the complex molecular machinery of these receptors and their role in the normal and pathological functioning of the brain, including the defined anatomical location of receptors functionally involved in specific physiological responses.

Therefore, the precise control over mGluR activity is crucial for a better understanding of their dynamics and function either in cells or in living organisms. Over the past few years, different optical methods have been developed to manipulate mGluRs with accurate spatial and temporal resolution via the use of light and photoactive molecules as external triggers for 
receptor activation or inactivation. A first approach, optogenetic pharmacology, is based on covalently attached light-operated ligands while the second one, photopharmacology (also known as optopharmacology), is based on freely diffusible light-operated ligands. The present article will review the recent development of these two optical methods and their in vitro and in vivo applications to study mGluRs.

\section{Optogenetic pharmacology}

Optogenetic pharmacology is based on tethered photocontrolable ligands that bind covalently to genetically modified proteins $[15,16]$. Once attached, these ligands enable rapid, reversible and reproducible photoactivation or photoinactivation in specific cells expressing their target (Fig. 1). Before being applied to mGluRs, this approach has been used to photocontrol various voltage-gated and ligand-gated ion channels [15].

Light-controlled mGluRs have been designed thanks to the joint efforts of Isacoff's and Trauner's labs. They developed a first generation of "LimGluRs" based on photoswitchable tethered ligands (PTLs) that contain a maleimide for cysteine attachment at one end, and connected via a photoisomerizable azobenzene linker to a glutamate derivative at the other end. These ligands, called MAGs, covalently bind to mutated mGluRs where a geometrically appropriate cysteine-attachment point has been incorporated. Azobenzene possesses a stable trans isomer and a metastable cis isomer [17]. Trans-to-cis isomerization of azobenzene can be induced by UV light illumination, while cis-to-trans isomerization can be induced by visible light or heat. Under illumination at appropriate wavelengths, azobenzene photoisomerization induces a change in the overall conformation of the MAG, enabling (or not) the interaction of the glutamate moiety with its binding pocket. Using this approach, several 
LimGluRs have been generated: light-agonized LimGluR2, LimGluR3 and LimGluR6 and a light-antagonized LimGluR2 [18]. LimGluR2 has been expressed in heterologous cells, in rodent brain slices and in vivo in zebrafish where it controlled the function associated with mGlu2 receptors by light [18]. To enable a more precise spatial control of LimGluRs, some MAGs have been modified to enable a more accurate spatial activation by 2-Photon techniques [19].

More recently, a second generation of light-controlled mGluRs has been developed using the SNAP-tag or CLIP-tag technology and photoswitchable orthogonal remotely tethered ligands (PORTL). In this method, the receptors are genetically modified to incorporate a SNAP-tag or a CLIP-tag at their N terminus. SNAP-tag [20] is a monomeric protein of 182 residues $(20 \mathrm{kDa})$, derived from the enzyme AGT, that recognizes and irreversibly binds to $\mathrm{O}^{6}$-benzylguanine (BG) derivatives. A SNAP derivative, called CLIP-tag, has been developed to specifically react with $\mathrm{O}^{4}$-benzylcytosine (BC) derivatives [21]. Since SNAP-tag and CLIP-tag possess orthogonal substrate specificities, SNAP- and CLIP-tagged proteins can be labeled simultaneously and specifically with different molecular probes in living cells. This has proven to be a highly useful and potent approach in life sciences for multiple applications ranging from protein detection in microscopy to the single molecule study of protein-protein interaction (see [22] for review). In the field of mGluRs, this technology has notably been used to study homodimerization [23], to discover specific heterodimeric mGluRs [24], to develop mGluRs biosensors [25,26] and to probe receptor activation dynamics at a single molecule level [27]. For optogenetic pharmacology, PORTLs have been designed to covalently bind to the SNAP tag or CLIP tag incorporated into the targeted receptor. They are composed of a glutamate moiety, followed by a long flexible linker containing an azobenzene and terminated with a BG or BC that specifically react with the SNAP tag or CLIP tag domains. This strategy has first been applied to mGlu2 permitting the photoactivation of 
mGlu2 and the control of excitability in heterologous cells or transfected neurons [28]. Then, combining SNAP and CLIP tagged receptors and specific spectral variants of PORTLs, Levitz and colleagues have created a family of light-gated group II/III mGluRs [29], allowing multiplexed orthogonal optical control within receptor homo or heterodimers.

\section{Photopharmacology}

Photopharmacology (also called optopharmacology) is based on freely diffusible, druglike, light-operated ligands [30,31]. It allows the control of the function of the ligand on its target by light. Contrary to optogenetic pharmacology, no genetic modification of the targeted receptor and its exogenous expression are required, enabling the photocontrol of endogenous receptors. Two types of photo-regulated drugs have been developed for photopharmacology: photoactivable ligands and photoswitchable ligands (Fig. 1).

\section{Photoactivable ligands}

Photoactivable ligands are inactive 'caged' compounds that can be photoactivated following brief pulses of light. They are constituted of a ligand linked to a photo-labile protecting group that will be removed following illumination, enabling the uncaged ligand to bind to its receptor (Fig. 1). Therefore, these photoactivable ligands enable the precise control of the onset of drug activity at a specific location. 
This method was developed during the seventies and first applied to nucleotides [32,33]. Subsequently, the caged strategy was adapted to different neurotransmitters, including glutamate and GABA [34,35]. Photoactivation of caged glutamate greatly improved the spatial and temporal resolution of synaptic connectivity mapping in neuronal networks [36]. It has also been used to study the function of mGluRs [37]. However, the use of caged glutamate is somehow limited due to the lack of subtype selectivity. This led to the development of iGluR and mGluR selective compounds.

Recently, the first photoactivable ligand selective for an mGluR subtype was designed [38]. This caged compound called JF-NP-26 is an inactive photo-caged derivative of raseglurant (ADX-10059), a negative allosteric modulator (NAM) of mGlu receptors. Illumination of JF-NP-26 with violet light induces a photochemical reaction prompting the release of the active ligand, raseglurant, and the coumarin DEACM, and thus the blockade of mGlus receptors activity was observed in vitro in cell-based assays or in vivo in animal models of pain [38].

\section{Photoswitchable ligands}

Photoswitchable mGluRs ligands are azobenzene-containing molecules that can rapidly and reversibly photo-isomerize under specific light, passing from an 'active' to an 'inactive' configuration, or vice versa (Fig. 1). Indeed, azobenzene $\mathrm{N}=\mathrm{N}$ double bond geometry changes during photo-isomerization [17], inducing a change in the configuration of the ligand which drastically affects the affinity for its target. In the dark or under white light, the azobenzene moiety is in a trans configuration while it reaches the cis configuration upon illumination at 
the adequate wavelength. Relaxation to the generally thermodynamically more stable transisomer can be induced by irradiation or by spontaneous thermal relaxation.

In the last few years, photoswitchable ligands have been developed for various ion channels and receptors, such as GABA $\mathrm{A}_{\mathrm{A}}$ [39] and NMDA receptors [40], $\mathrm{K}_{\mathrm{ATP}}$ [41], TRPA1 [42] or GIRK channels [43], and GPCRs such as adenosine [44], $\mu$-opioid [45] or cannabinoid receptors [46] among others.

Several freely diffusible photoswitchable ligands targeting mGluRs have been developed. The first of them was Alloswitch-1, an mGlu 5 receptor NAM [47], which was the first example of an allosteric photoswitchable ligand targeting a GPCR. Alloswitch-1 was developed based in the structure of VU0415374, an allosteric ligand of mGlu4 receptors [48] in which an azobenzene was formally inserted replacing an amido group. Illumination by green or violet light stabilizes either the trans- or the cis-configuration of the ligand which

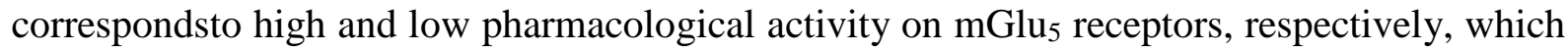
has been observed both in vitro or in vivo. Similarly, Optogluram is a photoswitchable mGlu4 positive allosteric modulator (PAM) [49] which allows for selective, reversible and repeated optical manipulation of mGlu 4 receptors activity with light both in vitro and in vivo. This ligand is also based in VU0415374 structure but with the azobenzene formally replacing a different amido group to that used in Alloswitch-1. The enhancement of mGlu 4 receptors activity observed by optogluram in the trans-form was reduced upon isomerization to the cisconfiguration after irradiation with violet light. The potentiation of $\mathrm{mGlu}_{4}$ receptors is recovered after green light Optogluram photoisomerization from cis to trans.

As the coefficient of light absorption by tissue depends on wavelengths and ultraviolet light which could potentially be damaging to irradiated tissues, it is interesting to design ligands that can photo-isomerize at red-shifted wavelengths. Recently, OptoGluNAM4.1, a 
blue light-sensitive mGlu$_{4}$ receptors photoswitchable NAM has been described [50]. Interestingly, after irradiation with blue light, this compound relaxes very fast from its cis to trans configuration, recovering the initial configuration by simply switching-off the illumination thus allowing the use of a single light source. This compound is active both in vitro and in vivo, being able to block the action of mGlu 4 receptor agonist in a light-dependent manner.

More recently, a series of photoswitchable mGlus receptor NAMs based on the phenylazopyridine scaffold present in Alloswitch-1 has been generated [51]. Most of the trans-isomers of these ligands are active both in vitro in cell-based assays and in vivo in zebrafish behavioral experiments. Interestingly, depending on their structures, the ligands exhibit different photo-isomerization properties with, notably, optimal illumination wavelengths varying from 360 to $500 \mathrm{~nm}$.

\section{Applications}

\section{In vitro applications}

In vitro, optogenetic pharmacology and photopharmacology have great potential in allow us to understand how receptors function at the molecular level, how they interact with each other and how they modulate the activity of a given cell. They can be of particular interest to study all of the kinetic aspects of receptor activation and function, which are impossible to be address by other means [52].

Firstly, light-controlled mGluRs that can be rapidly and repetitively switched on and off by light can be useful optogenetic tools to understand the activation mechanisms of mGluRs 
and their associated signaling. Using LimGluRs, Levitz and colleagues demonstrated the ability of LimGluR2 to optically modulate native downstream targets of mGlu2 receptors both in heterologous cells and in cultured hippocampal neurons [53]. This study also provides proof of concept that this strategy can be applied to study the synaptic activity of mGluRs in neural circuits. Indeed, the authors demonstrated that light-activation of LimGluR2 reduces excitability and inhibits neurotransmitter release in mouse brain slices [53]. Furthermore, the design of specific PTL's compatible with 2-photon microscopy will enable high-precision activation of LimGluRs, making them valuable tools to map the modulation of synaptic activity by these receptors in neuronal networks [19]. mGluRs are complex allosteric machines composed of two subunits each containing different modules which interact with each other during the activation process. Large conformational rearrangements occur upon activation to transmit the information from the extracellular domains binding glutamate to the transmembrane domains coupling G-proteins [6]. Photoswitch has been used to probe the receptor dynamics during activation, demonstrating the cooperativity between the subunits of an mGlu2 homodimer or an mGlu2/3 heterodimer [53]. The system has been improved to enable dual optical control of multiple receptors using bioorthogonal tethering of PORTL either to SNAP or CLIP tags $[28,29]$. Levitz and colleagues recently achieved the independent photoswitch of a single or the two subunits composing an mGluR homodimer or heterodimers, demonstrating that these optogenetic tools can provide subunit, spatial and temporal precisions to the study of mGluRs dynamics and function.

Photoswitchable and photoactivable ligands enable the optical control of endogenous receptors in their native environment. Photolytic release of raseglurant from its cagedderivative JF-NP-26 effectively induces the block of the activity of heterologously expressed mGlus receptors in HEK293 cells and native ones in striatal primary neurons [38]. Photoswitchable allosteric modulators of mGlu5 and mGlu4 receptors reversibly 
photoregulate the activity of their target in transfected HEK293 cells or in primary culture of neurons or astrocytes [47,49-51]. Contrary to optogenetic pharmacology, no genetic modification of the targeted receptor and its exogenous expression are required, enabling the photocontrol of endogenous receptors. Photoactivable ligands have been used for precise stimulation of receptors at defined locations in brain slices, allowing mapping of the distribution and function of neurotransmitter receptors in neuronal networks. Such an application could be feasible in principle using the photoactivable mGlu 5 receptors NAM JFNP-26 and the photoswitchable allosteric modulators of mGlu 4 and mGlu 5 receptors.

\section{In vivo applications}

Combining optogenetic pharmacology and photopharmacology with the high spatial and temporal resolution of optical manipulation offers the possibility to investigate the physiopathological function of receptors at precise locations in living animals. However, in vivo optogenetic pharmacology and photopharmacology face several challenges. The first one concerns the genetic manipulation required by optogenetics to express the modified receptor to allow anchoring of the remotely tethered photoswitchable ligands in the targeted organisms. However, this point can be adressed by the use of viral vectors [54]. The second challenge resides in local ligh delivery in living organisms. Indeed, although relatively easy with small animal models, such as tadpoles or zebrafish, or in superficial tissues or retina, light delivery is more challenging in deep tissues of bigger organisms, such as rodents. However, thanks to the advent of optogenetics, the development of technical solutions for optical control is in constant progress and overcomes this obstacle. 
The small size and transparency of Zebrafish and Xenopus tropicalis tadpoles allow easy and non-invasive optical manipulations in the presence of a photoresponsive molecule. Alloswitch-1, a photoswitchable NAM of mGlus receptors, allows photocontrol of the swimming behavior of the Xenopus tadpole [47]. Zebrafish, in particular, is a genetic model of choice since many years, enabling specific targeting of optogenetic tools in populations of cells and their subsequent monitoring by light [55]. The light-controlled mGluRs can be expressed in zebrafish. Photo-activation of LimGluR2 reversibly and repeatedly modulates the escape behavior in zebrafish [18]. Concerning photopharmacological applications, zebrafish also presents the interest of possessing mGluRs with high sequence homology with the ones of mammals [56], reducing the chances of having inactive ligands due to species selectivity. Notably, the allosteric binding pocket of mGlu 4 receptor is well conserved between rodents and zebrafish [50]. Photoswitchable allosteric modulators of $\mathrm{mGlu}_{4}$ and mGlu $_{5}$ receptors enable the photocontrol of the motility behavior of zebrafish larvae [50,51]. One of the striking attributes of the zebrafish model is the robustness of the translation of the photoswitchable properties of the ligands determined in cell-based assays with the ones observed in vivo, as illustrated in the study by Gomez-Santacana and colleagues [51]. In this structure-activity relationship study, the photopharmacological properties of a series of photoswitchable NAMs of mGlu $_{5}$ receptors have been determined both in vitro in heterologous cells and in vivo in zebrafish. The optimal wavelengths of illumination range from 360 to $500 \mathrm{~nm}$. The "photoisomerization scores" (PIS) which rank the effectiveness of the azobenzene photoisomerization (from poor to high) and the "photoinduced potency shifts" (PPS) which quantify the effectiveness of the compound photoswitching (corresponding to the ratio of the IC50's under illumination and in the dark) of most of the ligands are comparable both in vitro and in vivo [51]. 
Using photoswitchable [49] or photoactivable mGluR drugs [38], two pioneering studies have recently demonstrated the feasibility of controlling endogenous neuromodulatory mechanisms in the brain of freely moving mice. Zussy and colleagues have injected optogluram, a photoswitchable PAM of mGlu4 receptors, and photocontrolled the activity of native mGlu 4 receptors in a specific area of the brain of freely behaving mice. This study revealed that it is possible to regulate persistent pain-related symptoms in a temporally and spatially restricted manner taking control of amygdala mGlu 4 receptors by light with a photoswitchable PAM molecule and controlling the effects at a peripheral site. To our knowledge, this was the first study that established that photopharmacology with a small diffusible drug-like photoswitchable ligand can be used in vivo to regulate the behavior in a disease model. Another study has demonstrated the possibility to use photoactivatable

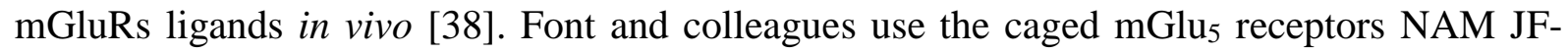
NP-26 to control inflammatory or neuropathic pain in mice by uncaging this compound either at the periphery, in the paw, or centrally in the thalamus. Strikingly, this study shows that photoreleasing the active drug at specific locations in the brain or in the periphery results in analgesia, whereas targeting other mGlus receptors-containing tissues is devoid of any effect on pain sensitivity, thus linking anatomically restricted mGlu$_{5}$ receptor antagonism to analgesic effects in vivo.

Finally, yet importantly, there have been two recent in vivo applications of optogenetics in the field of mGluRs. First, using a strategy known as opto-XR [57], Van Wyk and colleagues designed a chimeric receptor consisting of the intracellular domains of the ON-bipolar cellspecific mGlu 6 receptor and the light-sensing domains of melanopsin that they called OptomGluR6 [58]. This chimeric receptor responds to daylight by activating the GIRK signaling pathway in cells. Transgenic expression of opto-mGluR6 specifically in ON-bipolar cells by a viral approach and the GRM6/sv40 enhancer promoter restores vision in the blind rd1 mouse 
[58]. More recently, Berry and colleagues restored vision of blind rd1 mice by transgenically expressing the light-activated SNAG-mGluR2 [28] in retinal ganglion cells using an AAV vector containing the SNAP-mGlu2 receptors under control of the synapsin promoter [59]. These two strategies raise hope for future therapeutic applications to restore vision.

\section{Conclusion}

As compared to conventional pharmacological approaches, the use of light to trigger receptor responses is a potent and unique tool to study their mechanisms of action, and their physiological and biological roles, at cellular, tissue and living animal experimental levels. In particular, optogenetic pharmacology and photopharmacology can greatly improve spatial and temporal resolution of the control over their target protein. The fundamental research tools presented in this review are of particular interest to study the molecular and kinetic aspects of mGlu receptor activation. They also hold great promise for exploring the role and therapeutic potential of mGluRs and their associated signaling in physiology and disease. In the near future, the number of available photocontrolled tools will likely increase, expanding the range of the current applications and helping to understand the biological particularities of the mGluRs extraordinary molecular machines

\section{Acknowledgments}


The authors thank Ebba L. Lagerqvist for critical reading of the manuscript. We acknowledge financial support from the Agence Nationale de la Recherche (ANR-12-NEUR0003 and ANR-16-CE16-0010-01), the ERANET Neuron LIGHTPAIN project, the Fondation Recherche Médicale (FRM team DEQ20130326522), the Centre National de la Recherche Scientifique, the Spanish Ministry of Economy, Industry Competitiveness (SAF2015-74132JIN) and MINECO (CTQ2014-57020-R and CTQ2017-89222-R), cofinanced by the European Regional Development Fund (FEDER) and the Catalan Government (2014SGR109). 


\section{Bibliography}

1. Pin JP, Galvez T, Prezeau L: Evolution, structure, and activation mechanism of family 3/C G-protein-coupled receptors. Pharmacol Ther 2003, 98:325-354.

2. Flor PJ, Acher FC: Orthosteric versus allosteric GPCR activation: the great challenge of group-III mGluRs. Biochem Pharmacol 2012, 84:414-424.

3. Lindsley CW, Emmitte KA, Hopkins CR, Bridges TM, Gregory KJ, Niswender CM, Conn PJ: Practical Strategies and Concepts in GPCR Allosteric Modulator Discovery: Recent Advances with Metabotropic Glutamate Receptors. Chem Rev 2016, 116:6707-6741.

4. Nicoletti F, Bockaert J, Collingridge GL, Conn PJ, Ferraguti F, Schoepp DD, Wroblewski JT, Pin JP: Metabotropic glutamate receptors: from the workbench to the bedside. Neuropharmacology 2011, 60:1017-1041.

5. Julio-Pieper M, Flor PJ, Dinan TG, Cryan JF: Exciting times beyond the brain: metabotropic glutamate receptors in peripheral and non-neural tissues. Pharmacol Rev 2011, 63:35-58.

6. Pin JP, Bettler B: Organization and functions of mGlu and GABAB receptor complexes. Nature 2016, 540:60-68.

7. Kubo Y, Miyashita T, Murata Y: Structural basis for a Ca2+-sensing function of the metabotropic glutamate receptors. Science 1998, 279:1722-1725. 
8. Jiang JY, Nagaraju M, Meyer RC, Zhang L, Hamelberg D, Hall RA, Brown EM, Conn PJ, Yang JJ: Extracellular calcium modulates actions of orthosteric and allosteric ligands on metabotropic glutamate receptor 1alpha. J Biol Chem 2014, 289:1649-1661.

9. Kuang D, Hampson DR: Ion dependence of ligand binding to metabotropic glutamate receptors. Biochem Biophys Res Commun 2006, 345:1-6.

10. Vafabakhsh R, Levitz J, Isacoff EY: Conformational dynamics of a class C G-proteincoupled receptor. Nature 2015, 524:497-501.

11. DiRaddo JO, Miller EJ, Hathaway HA, Grajkowska E, Wroblewska B, Wolfe BB, Liotta DC, Wroblewski JT: A real-time method for measuring cAMP production modulated by Galphai/o-coupled metabotropic glutamate receptors. J Pharmacol Exp Ther 2014, 349:373-382.

12. Tora AS, Rovira X, Dione I, Bertrand HO, Brabet I, De Koninck Y, Doyon N, Pin JP, Acher F, Goudet C: Allosteric modulation of metabotropic glutamate receptors by chloride ions. FASEB J 2015, 29:4174-4188.

13. Niswender CM, Conn PJ: Metabotropic Glutamate Receptors: Physiology, Pharmacology, and Disease. Annu Rev Pharmacol Toxicol 2010, 50:295-322.

14. Acher F, Goudet C: Therapeutic potential of group III metabotropic glutamate receptor ligands in pain. Curr Opin Pharmacol 2015, 20C:64-72.

15. Kramer RH, Mourot A, Adesnik H: Optogenetic pharmacology for control of native neuronal signaling proteins. Nat Neurosci 2013, 16:816-823.

16. Leippe P, Koehler Leman J, Trauner D: Specificity and Speed: Tethered Photopharmacology. Biochemistry 2017, 56:5214-5220. 
17. Beharry AA, Woolley GA: Azobenzene photoswitches for biomolecules. Chem Soc Rev 2011, 40:4422-4437.

18••. Levitz J, Pantoja C, Gaub B, Janovjak H, Reiner A, Hoagland A, Schoppik D, Kane B, Stawski P, Schier AF, et al.: Optical control of metabotropic glutamate receptors. Nat Neurosci 2013, 16:507-516.

This article describes the development of the first light-controlled mGluRs using photoswitchable tethered ligands (PTL) binding to modified receptors, harboring a geometrically appropriate cysteine-attachment point. It describes photoactivable versions of mGlu2 and mGlu3 and photoinactivable versions of mGlu2 and mGlu6. LimGluR2 allows reversible photocontrol of synaptic transmission in hippocampal slices and modulate the acoustic startle response in zebrafish.

19. Carroll EC, Berlin S, Levitz J, Kienzler MA, Yuan Z, Madsen D, Larsen DS, Isacoff EY: Two-photon brightness of azobenzene photoswitches designed for glutamate receptor optogenetics. Proc Natl Acad Sci U S A 2015, 112:E776-785.

20. Keppler A, Gendreizig S, Gronemeyer T, Pick H, Vogel H, Johnsson K: A general method for the covalent labeling of fusion proteins with small molecules in vivo. Nat Biotechnol 2003, 21:86-89.

21. Gautier A, Juillerat A, Heinis C, Correa IR, Jr., Kindermann M, Beaufils F, Johnsson K: An engineered protein tag for multiprotein labeling in living cells. Chem Biol 2008, $15: 128-136$.

22. Reymond L, Lukinavicius G, Umezawa K, Maurel D, Brun MA, Masharina A, Bojkowska K, Mollwitz B, Schena A, Griss R, et al.: Visualizing biochemical activities in living cells through chemistry. Chimia (Aarau) 2011, 65:868-871. 
23. Maurel D, Comps-Agrar L, Brock C, Rives ML, Bourrier E, Ayoub MA, Bazin H, Tinel N, Durroux T, Prezeau L, et al.: Cell-surface protein-protein interaction analysis with time-resolved FRET and snap-tag technologies: application to GPCR oligomerization. Nat Methods 2008, 5:561-567.

24. Doumazane E, Scholler P, Zwier JM, Eric T, Rondard P, Pin JP: A new approach to analyze cell surface protein complexes reveals specific heterodimeric metabotropic glutamate receptors. FASEB J 2011, 25:66-77.

25. Doumazane E, Scholler P, Fabre L, Zwier JM, Trinquet E, Pin JP, Rondard P: Illuminating the activation mechanisms and allosteric properties of metabotropic glutamate receptors. Proc Natl Acad Sci U S A 2013, 110:E1416-1425.

26. Scholler P, Moreno-Delgado D, Lecat-Guillet N, Doumazane E, Monnier C, CharrierSavournin F, Fabre L, Chouvet C, Soldevila S, Lamarque L, et al.: HTS-compatible FRETbased conformational sensors clarify membrane receptor activation. Nat Chem Biol 2017, $13: 372-380$.

27. Olofsson L, Felekyan S, Doumazane E, Scholler P, Fabre L, Zwier JM, Rondard P, Seidel CA, Pin JP, Margeat E: Fine tuning of sub-millisecond conformational dynamics controls metabotropic glutamate receptors agonist efficacy. Nat Commun 2014, 5:5206.

28••. Broichhagen J, Damijonaitis A, Levitz J, Sokol KR, Leippe P, Konrad D, Isacoff EY, Trauner D: Orthogonal Optical Control of a G Protein-Coupled Receptor with a SNAPTethered Photochromic Ligand. ACS Cent Sci 2015, 1:383-393.

This article describes the design of second-generation light-controlled mGluRs using photoswitchable orthogonal remotely tethered ligand (PORTL). This strategy is applied to an mGlu2 receptor genetically modified to incorporate a SNAP tag. PORTL are composed of a 
glutamate moiety, followed by a long flexible linker containing an azobenzene and terminated with a BG that specifically react with the SNAP tag. Photoactivation of mGlu2 allows the control of excitability in heterologous cells or transfected neurons. This strategy opens the way to optical control of multiple receptors in the same preparation using different protein tags, such as SNAP or CLIP tags, and PORTL spectral variants.

29•. Levitz J, Broichhagen J, Leippe P, Konrad D, Trauner D, Isacoff EY: Dual optical control and mechanistic insights into photoswitchable group II and III metabotropic glutamate receptors. Proc Natl Acad Sci U S A 2017, 114:E3546-E3554.

This study describes the generation of a family of light-controlled group II/III mGluRs using the PORTL strategy in combination with the SNAP/CLIP tag technology. Several variants of light-controlled mGluR2, 3, 6, 7, and 8 are designed. The article provides an analysis of the mechanisms of photoswitch and discusses the advantages of the PORTL vs. PTL approaches.

30. Broichhagen J, Frank JA, Trauner D: A roadmap to success in photopharmacology. Acc Chem Res 2015, 48:1947-1960.

31. Velema WA, Szymanski W, Feringa BL: Photopharmacology: beyond proof of principle. J Am Chem Soc 2014, 136:2178-2191.

32. Engels J, Schlaeger EJ: Synthesis, Structure, and Reactivity of Adenosine Cyclic 3',5'Phosphate Benzyl Triesters. Journal of Medicinal Chemistry 1977, 20:907-911.

33. Kaplan JH, Forbush B, 3rd, Hoffman JF: Rapid photolytic release of adenosine 5'triphosphate from a protected analogue: utilization by the Na:K pump of human red blood cell ghosts. Biochemistry 1978, 17:1929-1935. 
34. Wieboldt R, Gee KR, Niu L, Ramesh D, Carpenter BK, Hess GP: Photolabile precursors of glutamate: synthesis, photochemical properties, and activation of glutamate receptors on a microsecond time scale. Proc Natl Acad Sci U S A 1994, 91:8752-8756.

35. Wieboldt R, Ramesh D, Carpenter BK, Hess GP: Synthesis and photochemistry of photolabile derivatives of gamma-aminobutyric acid for chemical kinetic investigations of the gamma-aminobutyric acid receptor in the millisecond time region. Biochemistry 1994, 33:1526-1533.

36. Callaway EM, Katz LC: Photostimulation using caged glutamate reveals functional circuitry in living brain slices. Proc Natl Acad Sci U S A 1993, 90:7661-7665.

37. Crawford JH, Wootton JF, Seabrook GR, Scott RH: Activation of Ca2+-dependent currents in dorsal root ganglion neurons by metabotropic glutamate receptors and cyclic ADPribose precursors. J Neurophysiol 1997, 77:2573-2584.

38••. Font J, Lopez-Cano M, Notartomaso S, Scarselli P, Di Pietro P, Bresoli-Obach R, Battaglia G, Malhaire F, Rovira X, Catena J, et al.: Optical control of pain in vivo with a photoactive mGlu5 receptor negative allosteric modulator. Elife 2017, 6.

This article describes a photoactivable version of raseglurant, a selective negative allosteric modulator of mGlu5. Photolytic release of raseglurant from its caged-derivative JFNP-26 inhibits the signaling of heterologous mGlu5 in HEK293 cells and of native mGlu5 in striatal primary neurons. In vivo, photoreleasing the active drug at specific locations of the pain neuraxis in the brain or in the periphery results in analgesia.

39. Stein M, Middendorp SJ, Carta V, Pejo E, Raines DE, Forman SA, Sigel E, Trauner D: Azo-propofols: photochromic potentiators of GABA(A) receptors. Angew Chem Int Ed Engl 2012, 51:10500-10504. 
40. Laprell L, Repak E, Franckevicius V, Hartrampf F, Terhag J, Hollmann M, Sumser M, Rebola N, DiGregorio DA, Trauner D: Optical control of NMDA receptors with a diffusible photoswitch. Nat Commun 2015, 6:8076.

41. Broichhagen J, Schonberger M, Cork SC, Frank JA, Marchetti P, Bugliani M, Shapiro AM, Trapp S, Rutter GA, Hodson DJ, et al.: Optical control of insulin release using a photoswitchable sulfonylurea. Nat Commun 2014, 5:5116.

42. Kokel D, Cheung CY, Mills R, Coutinho-Budd J, Huang L, Setola V, Sprague J, Jin S, Jin YN, Huang XP, et al.: Photochemical activation of TRPA1 channels in neurons and animals. Nat Chem Biol 2013, 9:257-263.

43. Trads JB, Burgstaller J, Laprell L, Konrad DB, de la Osa de la Rosa L, Weaver CD, Baier H, Trauner D, Barber DM: Optical control of GIRK channels using visible light. Org Biomol Chem 2016, 15:76-81.

44. Bahamonde MI, Taura J, Paoletta S, Gakh AA, Chakraborty S, Hernando J, Fernandez-Duenas V, Jacobson KA, Gorostiza P, Ciruela F: Photomodulation of G proteincoupled adenosine receptors by a novel light-switchable ligand. Bioconjug Chem 2014, 25:1847-1854.

45. Schonberger M, Trauner D: A photochromic agonist for mu-opioid receptors. Angew Chem Int Ed Engl 2014, 53:3264-3267.

46. Westphal M, Schafroth MA, Sarott R, Imhof M, Bold C, Leippe P, Dhopeshwarkar A, Grandner J, Katritch V, Mackie K, et al.: Synthesis of Photoswitchable Delta9Tetrahydrocannabinol Derivatives Enables Optical Control of Cannabinoid Receptor 1 Signaling. J Am Chem Soc 2017, 139:18206-18212. 
47. Pittolo S, Gomez-Santacana X, Eckelt K, Rovira X, Dalton J, Goudet C, Pin JP, Llobet A, Giraldo J, Llebaria A, et al.: An allosteric modulator to control endogenous G protein-coupled receptors with light. Nat Chem Biol 2014, 10:813-815.

48. Engers DW, Field JR, Le U, Zhou Y, Bolinger JD, Zamorano R, Blobaum AL, Jones CK, Jadhav S, Weaver CD, et al.: Discovery, synthesis, and structure-activity relationship development of a series of $\mathrm{N}$-(4-acetamido)phenylpicolinamides as positive allosteric modulators of metabotropic glutamate receptor 4 (mGlu(4)) with CNS exposure in rats. J Med Chem 2011, 54:1106-1110.

49••. Zussy C, Gomez-Santacana X, Rovira X, De Bundel D, Ferrazzo S, Bosch D, Asede D, Malhaire F, Acher F, Giraldo J, et al.: Dynamic modulation of inflammatory pain-related affective and sensory symptoms by optical control of amygdala metabotropic glutamate receptor 4. Mol Psychiatry 2016 in press.

This article describes the first example of deep brain optical control of native receptors in freely behaving mice using photopharmacology. Optogluram, an mGlu4 PAM, allows rapid and reversible alleviation of chronic pain related behavioral symptoms.

50• Rovira X, Trapero A, Pittolo S, Zussy C, Faucherre A, Jopling C, Giraldo J, Pin JP, Gorostiza P, Goudet C, et al.: OptoGluNAM4.1, a Photoswitchable Allosteric Antagonist for Real-Time Control of mGlu4 Receptor Activity. Cell Chem Biol 2016, 23:929-934.

This article describes the in vitro and in vivo photopharmacological properties of OptoGluNAM4.1, a blue light sensitive and fast relaxing mGlu4 photoswitchable NAM. Interestingly, this ligand rapidly recovers its trans 'active' configuration by simply switchingoff the illumination thus allowing the use of a single light source. 
51 • Gomez-Santacana X, Pittolo S, Rovira X, Lopez M, Zussy C, Dalton JA, Faucherre A, Jopling C, Pin JP, Ciruela F, et al.: Illuminating Phenylazopyridines To Photoswitch Metabotropic Glutamate Receptors: From the Flask to the Animals. ACS Cent Sci 2017, 3:8191.

This article presents a series of photoswitchable mGlu5 NAMs based on the phenylazopyridine scaffold of Alloswitch-1 with different photo-isomerization properties. Interestingly, the photoswitchable properties determined in vitro in cell-based assays are robustly translated in vivo in zebrafish behavioral experiments.52. Lohse MJ, Hofmann KP: Spatial and Temporal Aspects of Signaling by G-Protein-Coupled Receptors. Mol Pharmacol 2015, 88:572-578.

53••. Levitz J, Habrian C, Bharill S, Fu Z, Vafabakhsh R, Isacoff EY: Mechanism of Assembly and Cooperativity of Homomeric and Heteromeric Metabotropic Glutamate Receptors. Neuron 2016, 92:143-159..

This study demonstrates the ability of the light-controlled LimGluR2 to modulate downstream effectors of mGlu2 in heterologous cells and in cultured neurons and proves that this strategy can be applied to study the synaptic activity of mGluRs in neural circuits in mouse brain slices.

54. Lerner TN, Ye L, Deisseroth K: Communication in Neural Circuits: Tools, Opportunities, and Challenges. Cell 2016, 164:1136-1150.

55. Portugues R, Severi KE, Wyart C, Ahrens MB: Optogenetics in a transparent animal: circuit function in the larval zebrafish. Curr Opin Neurobiol 2013, 23:119-126. 
56. Haug MF, Gesemann M, Mueller T, Neuhauss SC: Phylogeny and expression divergence of metabotropic glutamate receptor genes in the brain of zebrafish (Danio rerio). $\mathrm{J}$ Comp Neurol 2013, 521:1533-1560.

57. Airan RD, Thompson KR, Fenno LE, Bernstein H, Deisseroth K: Temporally precise in vivo control of intracellular signalling. Nature 2009, 458:1025-1029.

58••. van Wyk M, Pielecka-Fortuna J, Lowel S, Kleinlogel S: Restoring the ON Switch in Blind Retinas: Opto-mGluR6, a Next-Generation, Cell-Tailored Optogenetic Tool. PLoS Biol 2015, 13:e1002143.

This study describes the restoration of vision in blind rd1 mice by transgenic expression specifically in ON-bipolar cells of a chimeric receptor consisting of the intracellular domains of the ON-bipolar cell-specific mGlu6 receptor and the light-sensing domains of melanopsin called Opto-mGluR6. These results raise hope for future therapeutic applications of mGluRbased optogenetic to restore vision.

59••. Berry MH, Holt A, Levitz J, Broichhagen J, Gaub BM, Visel M, Stanley C, Aghi K, Kim YJ, Trauner D, et al.: Restoration of patterned vision with an engineered photoactivatable G protein-coupled receptor. Nat Commun 2017, 8:1862.

In this study, Berry and colleagues restored vision of blind rd1 mice by transgenically expression of the light-activated SNAG-mGluR2 in retinal ganglion cells. These results raise hope for future therapeutic applications of mGluR-based optogenetic to restore vision. 


\section{Figure 1. Different strategies to photocontrol mGluRs.}

Structural model of a dimeric mGluR composed of Venus Flytrap (VFT), Cysteine Rich (CRD) and a seven transmembrane domain (7TMD) domain. A first subunit is in dark grey while the second one is in light grey. The orthosteric binding site is located between the two lobes of the VFT (yellow shadow) while most allosteric modulators bind in a pocket located the 7TMD (blue shadow). For clarity, the orthosteric and allosteric pockets are only displayed in a single subunit. Optical control of mGluRs can be achevied via two main strategies. The optogenetic pharmacology strategy is based on photoswitchable tethered ligands binding to a genetically modified mGluR, harboring either a geometrically appropriate cysteineattachment point which will covalently bind PTL (1) or a SNAP or CLIP tag which will anchor PORTL (2). PTL contain a glutamate derivative at one end (orange circle) connected via a photoisomerizable azobenzene linker (green or purple circle) to a maleimide for cysteine attachment at the other end. PORTL are composed of a glutamate moiety, followed by a long flexible linker containing an azobenzene and terminated with a BG that will react specifically with the SNAP tag (or BC in case of a CLIP tag). In this figure, a PORTL is binding to its SNAP tag fused to the $\mathrm{N}$ terminus of the light grey subunit. The photopharmacology strategy is based on freely diffusible photoactivable (3) or photoswitchable ligands (4) that target endogenous mGluRs. Photoactivable ligands, also called 'caged' ligands, are constituted of a ligand linked to a photo-labile protecting group that will be removed following illumination, enabling the uncaged ligand to bind to its receptor. Photoswitchable mGluRs ligands are azobenzene-containing molecules that can rapidly and reversibly photo-isomerize under illumination at specific wavelenghts, passing from an 'active' to an 'inactive' configuration, and vice versa. 


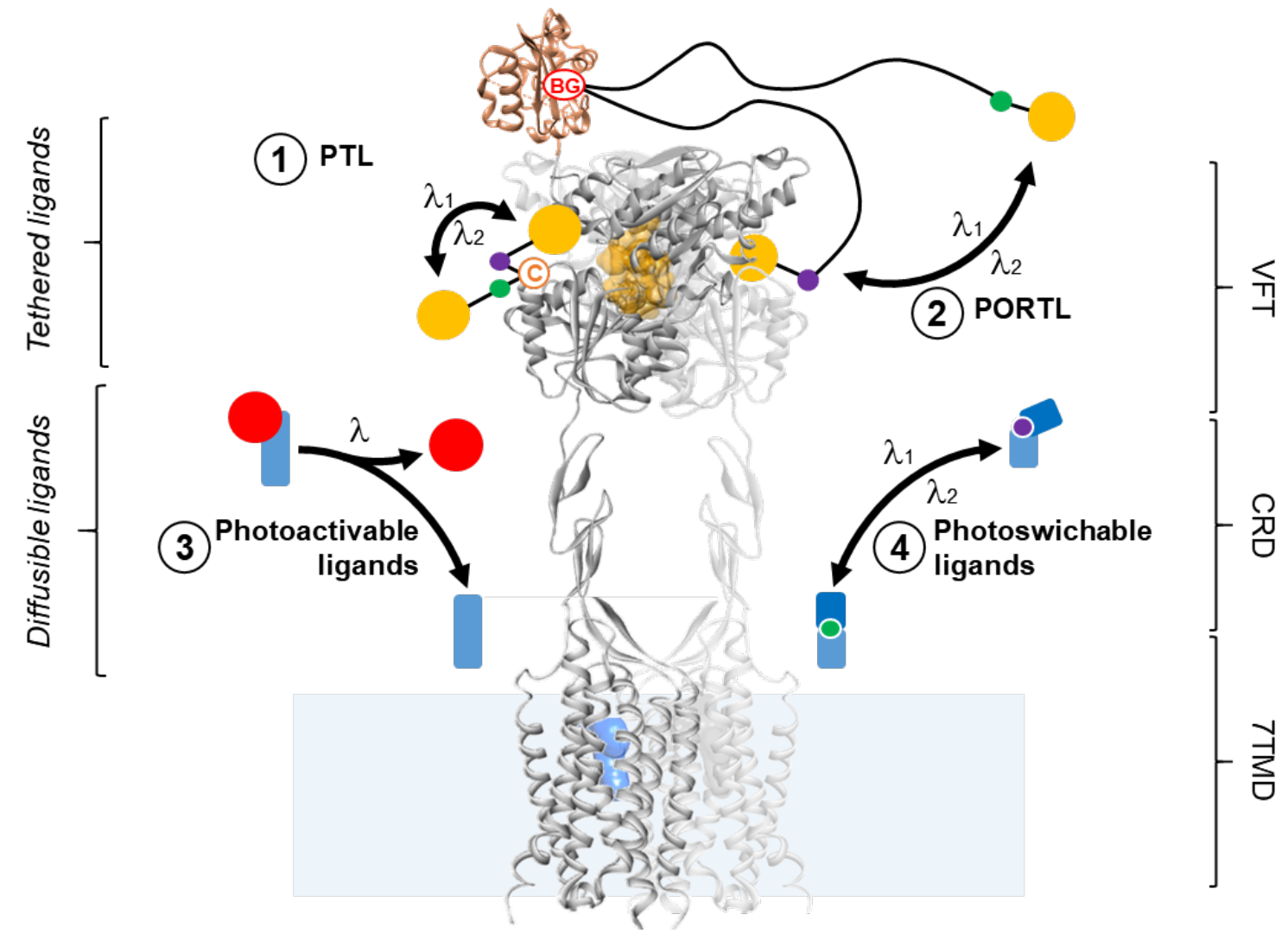

